\title{
Anti-Inflammatory Agents for Cancer Therapy
}

\author{
Elizabeth R. Rayburn ${ }^{1,2}$, Scharri J. Ezell ${ }^{1}$ and Ruiwen Zhang ${ }^{1,2,3^{*}}$ \\ ${ }^{1}$ Department of Pharmacology and Toxicology, Division of Clinical Pharmacology, ${ }^{2}$ Gene \\ Therapy Center, and ${ }^{3}$ Comprehensive Cancer Center, University of Alabama at Birmingham, \\ Birmingham, Alabama
}

\begin{abstract}
Inflammation is closely linked to cancer, and many anti-cancer agents are also used to treat inflammatory diseases, such as rheumatoid arthritis. Moreover, chronic inflammation increases the risk for various cancers, indicating that eliminating inflammation may represent a valid strategy for cancer prevention and therapy. This article explores the relationship between inflammation and cancer with an emphasis on epidemiological evidence, summarizes the current use of anti-inflammatory agents for cancer prevention and therapy, and describes the mechanisms underlying the anti-cancer effects of anti-inflammatory agents. Since monotherapy is generally insufficient for treating cancer, the combined use of anti-inflammatory agents and conventional cancer therapy is also a focal point in discussion. In addition, we also briefly describe future directions that should be explored for anticancer anti-inflammatory agents.
\end{abstract}

Keywords: Inflammation; Cancer; Carcinogenesis; Anti-Inflammatory agents; NSAID; COX-2 inhibitor; Corticosteroids; Chemoprotection; Pharmacokinetics; Chemosensitization

\section{Inflammation and Cancer}

It has long been recognized that infections and inflammation are related to cancer, and strong correlations between the presence of inflammation and the development of pre-cancerous lesions at various anatomic sites have been established. Exemplary studies have indicated that there is an approximately $14 \%$ increase in prostate cancer risk due to prostatitis ((1-3), a $25 \%$ increase in colorectal cancer risk due to ulcerative colitis $(4,5)$, and a $10^{-}$

Received 11/27/08; accepted 02/03/09

*Correspondence: Ruiwen Zhang, M.D., Ph.D., Department of Pharmacology and Toxicology, University of Alabama at Birmingham, VH 113, Box 600, 1670 University Blvd., Birmingham, AL 35294, USA.; Tel. 205.934.8558, Fax. 205.975.9330; email: ruiwen.zhang@ccc.uab.edu 20-fold increase in the risk of pancreatic cancer for patients who have experienced pancreatitis $(6-8)$. Thus, the presence of inflammation appears to induce or facilitate carcinogenesis.

That inflammation may lead to the initiation of cancer is reasonable considering that chronic inflammation is characterized by infiltration of mononuclear immune cells (including macrophages, lymphocytes, and plasma cells), tissue destruction, fibrosis, and increased angiogenesis (9, 10). Increased genomic damage, increased DNA synthesis, cellular proliferation, disruption of DNA repair pathways, inhibition of apoptosis, and the promotion of angiogenesis and invasion are also associated with chronic inflammation (11). All of these processes have been implicated in the initiation and progression of cancers. During chronic inflammation, pro-inflammatory molecules, such as cytokines, inducible nitric oxide synthase (iNOS), reactive oxygen species (ROS), and NF-kB are upregulated (12). Together, these processes provide a favorable microenvironment for the exponential growth of malignant cells. Thus, inflammation may provide both the key mutations and the proper environment to foster tumor growth.

There is extensive data demonstrating that inflammation plays a role in the establishment, progression, and/or aggressiveness of various malignancies. As a tumor develops, it expresses phenotypes similar to inflammatory cells (13). For example, numerous cancer cells express cytokines and chemokines and their receptors. These molecular mediators and their respective receptors have a significant impact on angiogenesis, cell migration, and metastasis $(14,15)$. In a study conducted by Chavey et al, a number of cytokines, including IL-6, IL-8, G-CSF (granulocyte colony stimulating factor), IFN-Y (interferon- ${ }^{-}$), and MIP-18 (macrophage inflammatory protein-18), were found to be more abundant in breast carcinoma than in normal breast tissue (16). Surprisingly, the expression level of IL-8, an important regulator of 
neutrophil activation and chemotaxis, and an activator of NF-kB, negatively corresponded with estrogen receptor status; the mediator was also more abundant in high-grade tumors than low-grade tumors, and was increased in tumors that exhibited high macrophage content and increased vascularization. MIP-18 expression was also higher in high-grade breast carcinomas compared to lowgrade tumors. Its expression corresponded to B lymphocyte, $\mathrm{T}$ lymphocyte, and macrophage infiltration, and was found to correlate with the overall presence of inflammatory cell components (16). Additionally, the observed levels of AP-1 (activator protein-1), a transcriptional target of NF$\mathrm{kB}$ and known regulator of numerous inflammatory cytokines, correlated with the expression levels of many of the examined cytokines.

\section{Targeting Inflammation for Cancer Therapy}

Given its myriad pro-tumor effects, inflammation has become a target for cancer prevention and therapy. $\mathrm{COX}^{-2}$ (cyclooxygenase 2, $P T G S 2)$ is the most frequently evaluated anti-cancer anti-inflammatory target, although numerous other targets, such as NF-kB, cytokines/cytokine receptors, chemokines/chemokine receptors, FGF/FGFR (fibroblast growth factor/receptor), and VEGF have also been examined (17-20). More than two decades ago, it was demonstrated that NSAIDs (nonsteroidal anti-inflammatory drugs) have anti-colon cancer effects $(21,22)$. Other clinical trials have indicated that long-term use of aspirin or other NSAIDs decreases the incidence of colorectal, esophageal, breast, lung and bladder cancers (23). While initial studies focused on various broadspectrum NSAIDs (which non-specifically inhibit both COX-1 and COX-2), more recent studies have examined COX-2 specific agents, such as celecoxib (24). However, given the GI toxicity and non-specific activity of NSAIDs (25), and the cardiotoxicity of specific COX-2 inhibitors, the use of such agents remains controversial.

Nevertheless, given that their toxicity is modest compared to conventional chemotherapeutic agents, various anti-inflammatory agents are still being investigated for cancer therapy and prevention. Many anti-inflammatory agents, including the NSAIDs, can alter the tumors themselves or the tumor microenvironment, potentially decreasing migration (26), increasing apoptosis (27), and increasing sensitivity to other therapies (28); thus, the agents still have immense promise against cancer. The remainder of this review will focus on the current status, mechanisms of action, and future directions for the use of anti-inflammatory agents for cancer therapy.

\section{Current Status of Anti-Inflammatory Agents for Cancer}

Dysregulated inflammation plays a major role in chronic illnesses, including diabetes, cardiovascular disease, arthritis, psoriasis, and cancer (29). As a result, there are numerous FDA-approved antiinflammatory agents for these indications. Although they were designed to decrease or prevent inflammation, many of these agents exhibit other properties such as anti-emetic, anti-thrombotic (30), anti-angiogenic (31), anti-proliferative, and proapoptotic activities (32), making them potential candidates for cancer therapy or prevention.

\section{a. NSAIDS}

Epidemiological data suggest that the incidence of breast, colorectal, and lung cancers is inversely related to the use of aspirin and nonsteroidal antiinflammatory drugs (33). Moreover, colorectal cancer patients who were long-term NSAID users had dramatically lower mortality rates than non-NSAID users (34). Randomized trials have demonstrated that aspirin can reduce the short-term risk of colon adenomas in patients with a prior history of adenomas (35). Another study demonstrated that the incidence and mortality from lung cancer for patients taking aspirin was significantly lower for non-smokers and former smokers than those who did not take aspirin (36). However, other studies, such as one in breast cancer patients, did not demonstrate any reduction in cancer risk for patients taking aspirin for up to 10 years follow-up (37). Although many clinical trials have not indicated any significant improvement for long-term prevention of different cancers (studies with $<10$ years follow-up), an examination of data from studies with longer follow-up periods demonstrated that aspirin use ( $300 \mathrm{mg} /$ day for $\geq 5$ years) can reduce the long-term risk of colon adenomas after a 10 year latency period (35).

Sulindac, another NSAID, has also been shown to decrease the recurrence and polyp number in FAP (familial adenomatous polyposis) patients (38) and lead to the regression of existing adenomas (39). Other NSAIDs, including ibuprofen and piroxicam, have also been shown to decrease the risk of developing cancers $(40,41)$. Thus, there appears to be a significant correlation between NSAID use and decreased incidence of primary and recurrent 
cancers, and decreased mortality, although the effects vary by dose, duration of exposure, cancer type, and the duration of follow-up. All of these agents have been approved by the FDA for different indications, and could readily be added to cancer therapy or prevention regimens.

\section{b. Specific COX-2 inhibitors}

Although the NSAIDs are effective antiinflammatory agents, newer agents were designed to specifically inhibit COX-2 in an effort to generate more active compounds with decreased GI toxicity. Early clinical studies involving patients with FAP demonstrated that celecoxib, a specific $\mathrm{COX}-2$ inhibitor, induced significant regression of existing adenomas (39). These findings led to the accelerated FDA approval of celecoxib for adjuvant therapy in the treatment of familial adenomatous polyposis in 1999 (42). Studies have also indicated that celecoxib may have activity against other cancers, such as ovarian cancer, as well (43). However, the use of celecoxib and other NSAIDs for cancer prevention and therapy remains controversial, with many clinical studies indicating that the compounds do not have significant beneficial effects for patients. Moreover, the side effects of the NSAIDs, ranging from moderate GI toxicity to cardiotoxicity, limit enthusiasm for their use, especially for cancer prevention. As a result of their cardiotoxicity, most of the COX-2 specific agents have been withdrawn from the market.

Despite the withdrawal of rofecoxib (Vioxx $\left.{ }^{\circledR}\right)$ and valdecoxib (Bextra ${ }^{\circledR}$ ) (as well as etoricoxib and parecoxib), there is still a high level of interest in celecoxib (Celebrex $\left.{ }^{\circledR}\right)$ in both the clinic and in preclinical studies. Celecoxib remains the only NSAID with FDA-approval for patients with familial adenomatous polyposis (FAP) (44) and is currently being investigated in a number of Phase II and III clinical trials both alone and in combination with other standard chemotherapy regimens (45). Given their toxicity and the controversy surrounding their cancer preventive and therapeutic affects, it is unlikely that COX-2 inhibitors will play a major role in cancer prevention or as monotherapy for cancer, however, they may represent effective treatments when used as adjuvants with other therapeutic approaches.

\section{c. Corticosteroids}

The corticosteroids, most commonly used to prevent or decrease the side effects of chemotherapy and radiation, have also shown anti-cancer activity when used alone or in combination with chemotherapeutic agents. For example, dietary administration of the glucocorticoid dexamethasone to $\mathrm{A} / \mathrm{J}$ mice exposed to tobacco smoke led to a more than $60 \%$ decrease in lung tumor incidence (46). Our studies have demonstrated that pre-treatment with dexamethasone can enhance the effects of conventional therapies against animal models of glioma, as well as breast, lung and colon cancers (47, 48). In fact, co-administration of dexamethasone led to a 2-4-fold increase in the efficacy of carboplatin and/or gemcitabine (47). A recent study in cell lines and a xenograft model demonstrated that dexamethasone treatment results in significant growth inhibition of renal cell carcinoma (49). It has also been demonstrated that dexamethasone can prevent the growth of estrogen-dependent breast cancer by antagonizing estrogen sulfotransferase (50). Other glucocorticoids, including hydrocortisone and prednisone, have also been shown to decrease the growth of cancer cells in culture and to decrease the growth of xenograft tumors in vivo $(51,52)$.

Thus, anti-inflammatory agents seem to have promise for the prevention or treatment of various human cancers. These agents, particularly the NSAIDs, have been documented to decrease migration (26), increase apoptosis (27, 53), and decrease angiogenesis $(54,55)$ of tumors. These effects, and epidemiological evidence of decreased cancer incidence and aggressiveness, prompted the numerous investigations of the potential of antiinflammatory agents for cancer prevention and therapy. While the results so far have been inconclusive, it cannot be denied that these compounds may represent novel, less toxic, agents for cancer therapy. However, like most of the agents currently used in the clinic, the anti-inflammatory compounds will likely need to be used as part of a combination regimen in order to achieve successful eradication of established tumors.

\section{Mechanisms of Anti-Cancer Action of Anti- Inflammatory Agents}

There is evidence that anti-inflammatory agents are effective adjuvants for conventional therapies $(28,56)$. Since monotherapy is typically insufficient to completely eradicate cancer, combination therapy is generally administered. A number of both clinical and pre-clinical studies suggest that the combined use of anti-inflammatory agents and conventional therapies may improve patient prognosis. Although the underlying mechanisms of action for the effects of anti-inflammatory agents as adjuvants are not 
fully demonstrated, three primary modes of action have been proposed: chemoprotection, alterations in pharmacokinetics or metabolism, and chemosensitization.

\section{a. Chemoprotection by anti-inflammatory agents}

There are a large number of adverse effects associated with the various clinically used anticancer agents. The majority of agents target rapidly proliferating cells, resulting in toxicity to both the tumor and various host tissues, primarily the gastrointestinal tract and bone marrow. Other toxicities arise as a result of accumulation of the agent in a particular anatomic region (e.g. the cardiovascular system or liver). These side effects limit the dose of agent that can be given, and greatly reduce patient quality of life. The ability to prevent or ameliorate these side effects would both improve the therapeutic response and improve patient quality of life.

There have been several studies that have demonstrated that administration of antiinflammatory agents can decrease the toxicity of conventional chemotherapeutic agents. For example, combining celecoxib with docetaxel decreased hematologic toxicity in patients with refractory metastatic prostate cancer, even though it only slightly decreased the pain index for patients (57). Similarly, the addition of celecoxib to a FOLFIRI (folinic acid, fluorouracil and irinotecan) regimen decreased the incidence of diarrhea for patients, allowing for administration of higher doses of irinotecan $\left(200 \mathrm{mg} / \mathrm{m}^{2}\right)$ than can typically be tolerated (58). A trial investigating the combination of capecitabine and celecoxib for patients with metastatic breast cancer indicated that the addition of the COX-2 inhibitor decreased the incidence of capecitabine-associated diarrhea and hand-foot syndrome (59). The recently completed GECO (Gemcitabine-Coxib) study was designed to evaluate the addition of rofecoxib to first-line chemotherapy regimens in patients with advanced non-small cell lung carcinoma (NSCLC). Despite the withdrawal of rofecoxib and the consequent cessation of the treatment arm, patients receiving adjuvant rofecoxib therapy exhibited higher response rates. Additionally, patients who had undergone at least 3 months of rofecoxib treatment experienced improved quality of life, measured by decreased fatigue, weight loss, pain, and analgesic consumption (32).

Our own studies have focused on the antiinflammatory glucocorticoid, dexamethasone. Dexamethasone has been used as an anti-emetic for cancer patients for many years, but has more recently been examined for potential chemoprotective and therapeutic effects. In our studies, we observed that dexamethasone decreased the hematotoxicity of gemcitabine and carboplatin in both CD-1 mice and human NSCLC patients in a dose- and schedule-dependent manner (60, 61). A study in advanced colorectal cancer patients demonstrated that administration of a different glucocorticoid, budesonide, led to a trend toward decreased incidence and duration of diarrhea in patients receiving irinotecan, as well as a diminished need for loperamide rescue (62).

Other NSAIDS, aspirin in particular, may also prove to be beneficial as anti-thrombotic agents if administered in combination with chemotherapeutic modalities. Approximately $90 \%$ of cancer patients display hypercoagulability, indicating that $\mathrm{co}^{-}$ administration of anti-thrombotic NSAIDs may aid in preventing arterial thromboses, allowing cytotoxic agents to more easily reach microscopic tumor foci and improve the prognosis of cancer patients (30)

Anti-inflammatory agents may also protect against neurotoxicity by decreasing or inhibiting the disruption of the blood-brain barrier. The cyclooxygenase (I and II) inhibitor indomethacin and COX-1 inhibitor, VAS, prevented disruption of the blood-brain barrier in rats following intracerebral injection of TNF- $\alpha$. These effects were attributed to both COX inhibition and a decrease in the expression and activity of matrix metalloproteinases (MMPs) 3 and 9 (63). Glucocorticoids, including dexamethasone, have also been shown to stabilize the blood-brain barrier, and are frequently used to treat cerebral edema. Dexamethasone may exert its effect by decreasing VEGF and increasing angiopoietin-1 (64).

These studies indicate that the use of antiinflammatory agents may prevent or inhibit many of the dose-limiting toxicities of several of the most commonly used anti-neoplastic agents. Anecdotal evidence suggests that other agents may have similar effects, implying that combination therapy with conventional chemotherapeutic agents and anti-inflammatory agents is an under-investigated area that may yield significant improvements in patient care.

\section{b. Alterations in pharmacokinetics or metabolism}

One of the mechanisms responsible for the chemoprotection induced by anti-inflammatory agents is that their administration can change the pharmacokinetics and pharmacodynamics of other 
therapeutic agents. For example, dexamethasone was shown to protect mice from hematotoxicity in a dose- $^{-}$and schedule-dependent manner. It was demonstrated that the drug altered the pharmacokinetics of carboplatin, gemcitabine and doxorubicin in both $\mathrm{CD}^{-1}$ mice and in a variety of mouse xenograft cancer models $(47,48,60)$. While there were no major differences in the plasma pharmacokinetics of carboplatin or gemcitabine in mice pre-treated with dexamethasone, there were significant decreases in their uptake by the spleen and bone marrow, accompanied by decreases in AUC (area under the curve), $\mathrm{T}_{1 / 2}$ (half-life) and $\mathrm{C}_{\max }$ (maximum concentration), as well as an increase in clearance of the drugs (47). More importantly, when animals were pre-treated with dexamethasone, the AUCs of the chemotherapeutic agents in the tumor were increased, while the AUCs in the bone marrow and spleen decreased (60). Similar effects were observed when dexamethasone was combined with adriamycin in a syngeneic model of mammary cancer (48). Thus, administration of the glucocorticoid can alter the pharmacokinetics of chemotherapeutic agents to decrease their toxicity and increase their activity in the tumor.

Even when the anti-inflammatory agents do not affect the pharmacokinetics of the chemotherapeutic agent, they may alter its metabolism, leading to differences in the concentration, half-life, and clearance of the active metabolite, thus altering the toxicity and efficacy of the agent(s). For example, several agents, including rofecoxib and mefenamic acid, are potent inhibitors of CYP1A2 (65). Dexamethasone is an inducer of CYP2D6, while celecoxib inhibits the activity of the enzyme (66). It is possible that by affecting CYP2D6 activity, dexamethasone or celecoxib may alter the efficacy of tamoxifen (which is metabolized by CYP2D6) treatment for preventing breast cancer recurrence. Other mechanisms by which the anti-inflammatory agents can alter metabolism of chemotherapeutic agents are also possible. Another NSAID, diclofenac, inhibited the metabolism of the novel chemotherapeutic agent DMXAA in mice by preventing its glucuronidation, leading to increased plasma AUC and decreased clearance (67).

Another factor influencing the amount of drug reaching the tumor is the tumor interstitial fluid pressure (IFP). Most tumors have high IFP, most likely resulting from abnormal vasculature or lymph vessels, or fibrosis of the surrounding stroma (68). This high pressure acts as a barrier to drug delivery to the tumor from the circulation, preventing therapeutic agents (most of which have relatively high molecular weights) from entering tumor cells (68). Various studies have demonstrated that high IFP correlates with a worse prognosis and decreased response to therapy (69). Several approaches have been shown to lower IFP, including VEGF and PDGF antagonists and dexamethasone (68). In our studies, we observed an increased uptake of chemotherapeutic agents by xenograft tumors following pre-treatment with dexamethasone (47). This effect may have been due to the change in tumor IFP (70). Another study indicated that there was a trend toward reduced tumor IFP in patients treated with celecoxib (71).

Thus, changes in the distribution or metabolism of chemotherapeutic agents can be induced by prior or co-administration of anti-inflammatory agents. While these changes can be deleterious under many conditions, it is also possible (as in the case of dexamethasone) to use these changes to protect normal tissues from toxicity or to increase the efficacy of a particular chemotherapeutic agent. Special care must be taken for studies that target differences in metabolism to ensure that all patients have metabolic enzymes that function at normal levels. Polymorphisms in the cytochrome p450 enzymes are common, and may lead to unexpected changes in toxicity or efficacy of both chemotherapeutic and anti-inflammatory agents.

\section{c. Chemosensitization by anti-inflammatory agents}

Beyond their ability to prevent or decrease patient toxicity, anti-inflammatory agents may also have therapeutic effects when combined with conventional agents, acting additively or synergistically, or sensitizing cancer cells to treatment with conventional cancer therapies. A large number of pre-clinical studies have examined the effects of celecoxib and other agents to further examine their ant-cancer efficacy and to determine potential mechanism(s) of action. For example, a recent study by Ponthan et al demonstrated that celecoxib induced additive or synergistic cytotoxic effects in vitro when combined with doxorubicin, etoposide, irinotecan or vincristine. When celecoxib was administered with irinotecan or doxorubicin to rats bearing neuroblastomas, there was a dosedependent decrease in tumor growth (72). Similarly, celecoxib significantly increased the cytotoxicity of docetaxel and another anti-cancer drug, COL-3, in prostate cancer cells, and acted synergistically with docetaxel in a xenograft model of prostate cancer (73). In a different study, celecoxib and another 
COX-2 inhibitor, SC-236, led to an enhanced response of syngeneic sarcomas and a xenograft model of glioma to radiation (30Gy, single dose) (74). The addition of celecoxib also increased the response of the tumors to docetaxel alone as well as to chemoradiation (docetaxel +10 Gy radiation) in a xenograft model (74).

In our studies, we observed that administration of dexamethasone increased the anti-tumor effects of carboplatin and gemcitabine in xenograft models of glioma as well as colon, lung, and breast cancers (47). Treatment with dexamethasone also increased the effects of doxorubicin in a syngeneic model of mammary cancer (48). While the changes in pharmacokinetics induced by dexamethasone may be largely responsible for their protection of normal host tissues, the compound also exerts its effect via another mechanism in the bone marrow and spleen. As part of its function as an anti-inflammatory and immunosuppressive agent, dexamethasone inhibits the production of various cytokines, decreasing the production and proliferation of lymphocytes. Since most chemotherapeutic agents (and radiotherapy) target rapidly proliferating cells, this preserves the cells in the bone marrow and spleen from the damage they would normally receive. For the same reason (inhibition of proliferation), dexamethasone can also be used to treat hematological cancers.

Beyond their obvious effects on cyclooxygenases, NSAIDs may act directly on solid tumors, decreasing their proliferation or increasing their apoptosis by a number of different pathways. An excellent review on these topics was recently published by N.R. Jana (27). Many studies have suggested that the anticancer activity of a number of NSAIDs, including aspirin, sulindac and indomethacin, may be dependent upon apoptosis brought about by their effects on NF-kB (RelA) signaling $(75,76)$. This decrease is not only due indirectly to the inhibition of cyclooxygenases, but also to a variety of other mechanisms. By sequestering the transcription factor in the nucleolus, the compounds can effectively decrease the expression of various NF- $\mathrm{kB}$ targets, including cell adhesion molecules, growth factors, and anti-apoptotic proteins (76). Another study suggested that the increase in the efficacy of doxorubicin by celecoxib was due to its suppression of NF-kB activity (which is generally upregulated by doxorubicin) (77). Although it acts by increasing the expression of $\mathrm{IkB}$, rather than sequestering NF- $\mathrm{kB}$ in the nucleolus, dexamethasone also inhibits NF- $k B$ activity, and this has also been suggested to play a role in its anti-cancer activity (78).
Other studies have shown that antiinflammatory agents increase apoptosis in cancer cells either by up-regulating pro-apoptotic molecules or down-regulating anti-apoptotic ones, or by activating the apoptotic pathway via other mechanisms. Dandekar et al demonstrated that celecoxib may additively or synergistically increase the cytotoxicity of docetaxel and the chemotherapeutic agent COL-3 by stimulating caspase activation, PARP cleavage, increasing the expression of $\mathrm{Bad}$, and decreasing the expression of XIAP (73). Aspirin also apparently exerts its effects on apoptosis at least partially through activation of caspases and pro-apoptotic proteins (79).

Various other anti-apoptotic and antiproliferative effects have been observed for the different anti-inflammatory agents. Numerous targets have been cited, including p27, p21, p53, E2F, ATM, ERK/MAPK, $\beta$-catenin, and a variety of others $(27,80-82)$. Inhibition of the proteasome, which also leads to down-regulation of NF-kB activity, may also be a mechanism underlying the pro-apoptotic activity of some of the compounds, particularly aspirin (83).

A study by Dittmann et al suggests that celecoxib enhances the radiosensitivity of cancer cells by inhibiting EGFR-mediated radioresistance, which is independent of the inhibition of COX-2 (84). In fact, many of the effects of the different compounds may occur independently of their effects on cyclooxygenase activity. Various investigators are now developing novel drugs based on the NSAIDs that lack their conventional COX-1/2 inhibitory effects, but still retain anti-cancer activity $(25,85)$.

Similar to the changes in tumor uptake brought about by the decrease in IFP, NSAIDs have been shown to increase uptake and enhance retention of therapeutic agents by tumors by inhibiting the expression or activity of drug resistance proteins. For example, sulindac inhibits the removal of chemotherapeutic agents from tumor cells by the multi-drug resistance protein 1 (MRP-1), and COX-2 inhibitors (indomethacin, nimesulide and naproxen) decrease the expression and activity of $\mathrm{P}$ glycoprotein (P-gp) $(86,87)$. However, a recent study by Gradilone et al demonstrated that celecoxib induces the overexpression of multidrug resistance proteins MRP4 and MRP5, leading to decreased in vitro efficacy of chemotherapeutic agents (88). Thus, the exact effects of the different agents and their use in combination with particular therapies require further examination. 
These examples illustrate the potential of antiinflammatory agents as adjuvant therapies. Pretreatment or co-administration of these agents can lead to chemoprotection, improving patient quality of life, increasing the MTD (maxiumum tolerated dose) of conventional chemotherapeutic agents, and protecting normal host tissues from damage by chemotherapeutic agents. Combining an antiinflammatory agent with conventional therapy can also lead to enhanced efficacy, due to alterations in distribution or metabolism, or due to additive or synergistic effects with these therapies.

As illustrated by their numerous mechanisms of action in protecting normal cells, altering the distribution or metabolism of therapeutic agents, or acting together with therapeutic agents (depicted in Figure 1), the effects of anti-inflammatory agents on normal and malignant tissues are diverse and complex. It may be necessary to either fine-tune the agents so that the most effective mechanism is targeted, or to design "multi-purpose" agents that capitalize on the complexity and apparent promiscuity of the compounds in order to prevent the escape of tumor cells from therapy. New clinical and pre-clinical trials are investigating a variety of novel anti-inflammatory drugs in the search for a more effective agent for use as monotherapy or as a chemoprotectant or chemosensitizer to other therapies. In addition, new combinations, new regimens (with respect to timing and dosing), and new points of intervention are also being tested.

\section{Future Directions}

Much emphasis is being placed on investigating new combinations and new regimens for using the anti-inflammatory agents. There are currently at least 98 active clinical trials investigating the use of celecoxib for cancer, most of which are focused on its use in combination with different chemotherapeutic agents and/or radiation therapy (45). These trials have the potential to rapidly affect patient care, since both the anti-inflammatory and anti-cancer agents/approaches have already been approved for use by the FDA. As such, there are several directions that researchers need to take in order to take advantage of the approval status of these agents.

\section{a. Pre-clinical screening}

Although there have been numerous pre-clinical studies (prior to the current clinical studies) of celecoxib in combination with various other approaches, the vast majority of NSAIDs and other anti-inflammatory agents have not been examined for possible chemoprotective or chemosensitizing effects when given in combination with other agents. There are numerous case studies and anecdotes in the literature that suggest potentially beneficial combinations of agents that can be tested in preclinical studies. While evaluations of changes in pharmacokinetics and metabolism require whole animals (or isolated organs), in vitro assays can be set up to determine whether the various compounds might act additively or synergistically with chemotherapeutic agents. Conventional studies of cell viability, proliferation, cell cycle progression, and apoptosis using MTT/MTS, BrdUrd incorporation, cell staining/flow cytometry and Annexin V/PI staining/flow cytometry, can be used (respectively) to assess whether an antiinflammatory agent can increase the effects of a conventional anti-cancer therapy. Migration assays or microvessel formation studies may also be of interest to determine whether the combination might have an effect on metastasis or angiogenesis. Special precautions should be taken to evaluate different sequences of exposure to the agents (pretreatment/post-treatment or simultaneous exposure), and the testing should be restricted to physiologically-relevant concentrations.

While studies involving animals must have a strong rationale given their expense and importance, they can be used to confirm any potential additive or synergistic effects observed in vitro. Mice bearing xenograft or syngeneic implants, or transgenic rodents, can be used to monitor tumor growth, metastasis and angiogenesis. Normal or tumorbearing animals can also be used to assess changes in the pharmacokinetics, pharmacodynamics, and tumor IFP, as well as the toxicity of the agents following different sequences of administration. Studies in other animals (e.g. dogs, primates) will be necessary prior to human clinical trials, but mice and rats can be used to demonstrate the proof-ofprinciple for novel combinations.

\section{b. Clinical studies}

When successful pre-clinical studies are translated to the clinic, there are also a number of improvements that can be made. First, it may be necessary to tailor treatments to a specific type of cancer, for example, hematopoeitic cancers versus solid tumors, or hormone sensitive versus resistant cancers. Leukemia and lymphoma may represent favorable cancers to study because the direct effects on cancer cells themselves can be measured in a 


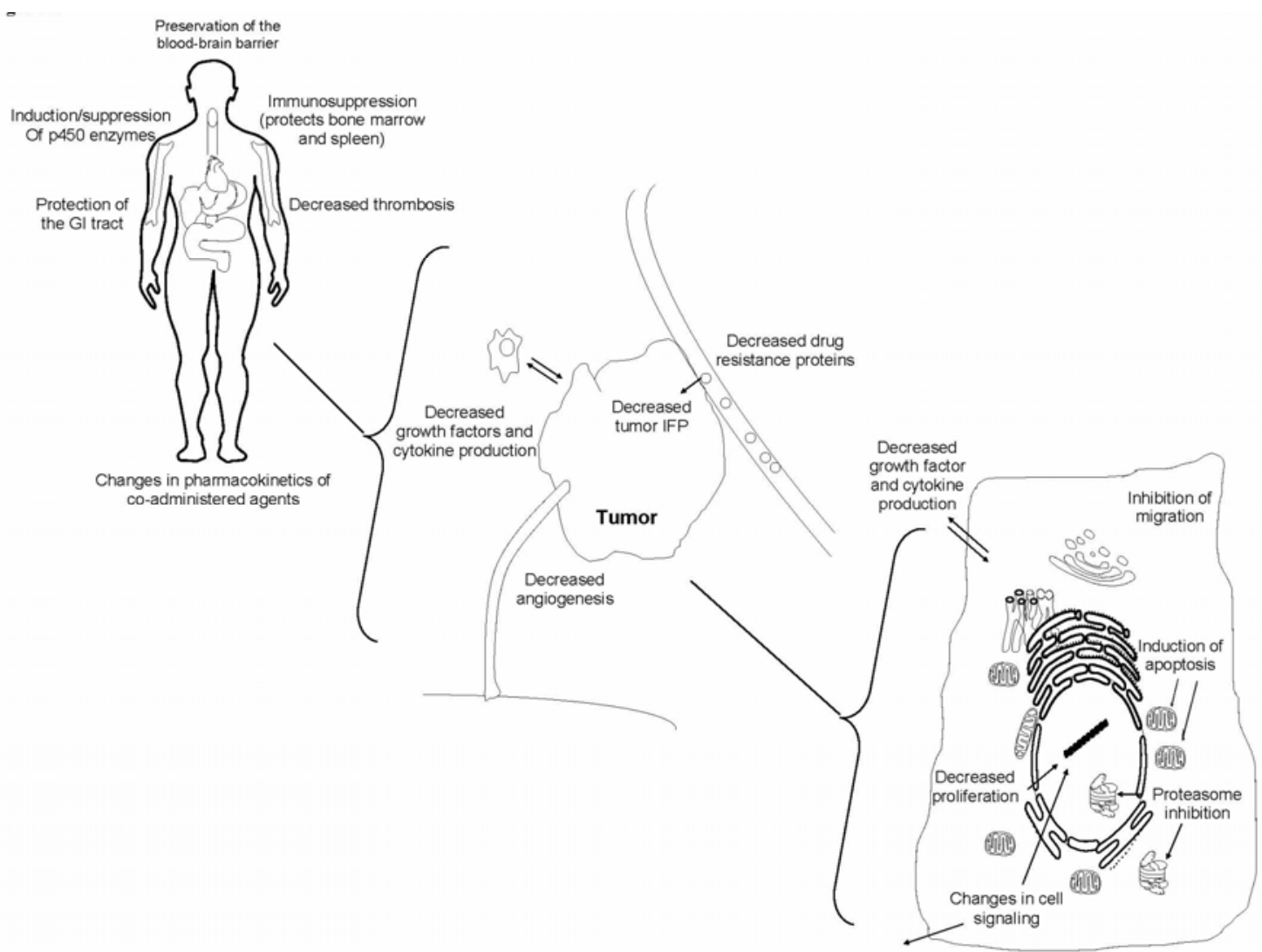

Figure 1. Schematic representation of the possible mechanisms of action for anti-inflammatory agents as chemoprotectants or as chemosensitizers to conventional cancer therapies. At the body or whole-organ level, anti-inflammatory agents can protect organs or specific sites within the body (e.g. bone marrow) against cytotoxic therapies, or can alter the uptake of cytotoxic agents within these sites. In the tumor itself, anti-inflammatory agents can allow for enhanced uptake/decreased exclusion of the cytoxic agent, or can interfere with tumor growth. At the cellular level, anti-inflammatory agents can decrease cancer cell proliferation, alter cell signaling, inhibit migration, or induce cell death.

less-invasive manner (in contrast to more invasive biopsies), and issues with tumor IFP and delivery to the tumor are minimized. Additionally, for other cancer types, tumor-specific serum biomarkers should be used to monitor the effects on the tumor whenever possible. Additional emphasis should be placed on determining surrogate markers for response that can be easily obtained through the blood. Some of these are already used in the clinic (e.g. PSA for prostate cancer), but other markers, such as ECPKA (extracellular calcium-dependent protein kinase), can be used for other cancers (89, 90).

As with the pre-clinical studies, it may be necessary to compare different dosing regimens. While the pre-clinical studies should establish the sequence of administration, the timing in human patients to obtain an optimal response will need to be determined, as will optimal doses and dosing frequencies and the duration of treatment/number of cycles of both the anti-inflammatory and other agents.

The transition from pre-clinical investigation to human clinical use can be very rapid. In our own experience, it took less than three years to transition from our pre-clinical studies to using dexamethasone to try to improve patient outcome (in terms of hematotoxicity) in the clinic (47, 61). However, dexamethasone has actually been used in the clinic for more than 50 years, and has been used in cancer patients for more than 40 years, which greatly facilitated the transition of the agent for a novel clinical use. Since there are a large number of anti-inflammatory and anti-cancer agents already approved for human use, and large quantities of data already exist about their toxicity, 
pharmacokinetics and side effects as single agents, their use in pre-clinical and clinical trials can advance rapidly. With concerted efforts from basic scientists and clinicians, there could be a dramatic change in the standard of treatment for cancer patients within 5-10 years. Nevertheless, there are a few detractors from using many of the currently available anti-inflammatory agents, including some undesirable toxicities (e.g. the cardiotoxicity of the COX-2 inhibitors) that have prompted the design and synthesis of a number of new classes of antiinflammatory agents that may eventually represent anti-cancer anti-inflammatory agents. Given the moderate toxicities of some of the existing FDAapproved agents, and a lack of enthusiasm for using anti-inflammatory agents for cancer based upon previous cancer prevention trials, some of these alternative agents in the pipeline may also eventually be used in the cancer field.

\section{c. In the pipeline}

The agents currently in pre-clinical and clinical development to prevent or decrease inflammation are diverse. They have mechanisms of action that differ from most of the currently used agents. While there are other agents under development, some of the most important classes are described briefly below.

\section{i. Anti-cancer agents modeled after COX-2 inhibitors}

A number of studies have suggested that the anti-tumor activity of COX-2 inhibitors is at least partly unrelated to their inhibition of the cyclooxygenase $(96,97)$. Thus, it may be possible to eliminate the toxicity of coxibs, making it possible for new, related agents to exert anti-tumor activity while avoiding COX-2 related side effects (GI toxicity and cardiotoxicity). In fact, several novel agents modeled after COX-2 "specific" drugs have been designed that do not inhibit COX-2. These have been shown to exert anti-cancer activity through various mechanism(s) of action, including induction of anoikis, inhibition of carbonic anhydrases, inhibition of the PDK1/Akt pathway, inhibition of MAPK or Stat3 signaling, or by causing cell cycle arrest $(25,44,98)$. While still in pre-clinical development, preliminary studies in cell lines and animal models of cancer suggest that these nonCOX-2 drugs based on celecoxib (e.g. 2,5-dimethylcelecoxib and OSU03012) have potent effects against cancer, and may represent the next generation of cancer preventive and/or therapeutic agents $(97,98)$.

\section{ii. NO-donating NSAIDs}

Another class of agents related to conventional anti-inflammatory compounds is the $\mathrm{NO}$-donating NSAIDs. Originally developed to prevent or counteract the GI toxicity of NSAIDs, the nitroxybutyl-ester or nitrosothiol NSAID derivatives have been synthesized so that the active component is still released to exert its anti-inflammatory actions, while the release of NO stimulates mucous secretion by the GI tract, protecting it from damage by the active agent (99). NO-donating versions of various NSAIDs have been synthesized, including diclofenac, naproxen, aspirin, sulindac, ibuprofen and indomethacin (100-102). Given the anti-cancer activities of the parent compounds, it is no surprise that many of these NO-donating NSAIDs have been shown to exert anti-cancer activities. In pre-clinical studies, various NO-donating NSAIDs have been demonstrated to induce apoptosis, inhibit the proliferation of, and induce cell cycle arrest in various human cancer cell lines (102). Animal studies have indicated that NO-NSAIDs can be used to prevent carcinogenesis in the min mouse and a model of pancreatic cancer, and can decrease the growth of xenograft tumors (103). In fact, these studies suggest that the NO-releasing compounds are more effective than their parent drugs (103). This may be due to possible anti-cancer effects of NO itself, or generation of different active metabolites following the removal of the leaving group (103). While results have not been released yet, a phase I study of NCX4016 (NO-acetylsalicylic acid) for preventing colorectal cancer was recently completed (clinicaltrials.gov). Rigas et al reported that this study was terminated prematurely following concerns about genotoxicity of the compound. Further examination of the compounds in animal models will demonstrate whether the NO-releasing agents are safe, but particular care will need to be taken to ensure that the benefits of prevention or therapy with NO-releasing agents outweigh the risks of future/secondary cancers.

\section{iii. Natural products}

While various plants and plant extracts have been used for millennia in Ayurvedic and Chinese medicine, their use is now being investigated as a complementary or alternative approach to conventional therapies in westernized countries. A large number of foods and natural compounds have been found to have anti-inflammatory properties, including chili peppers (capsaicin), grapes/red wine (resveratrol), garlic (various compounds), curry powder (curcumin), ginseng (ginsenosides), as well 
as numerous other fruits, vegetables and herbs (104107).

Natural products have been shown to prevent or decrease inflammation through a variety of mechanisms, including inhibition of NF- $\mathrm{kB}$, COX-1 and -2 , and MAPK, JNK and ERK1/2 signaling, as well as decreasing VEGF and iNOS (104-107). Many of these agents also exert anti-proliferative, pro-apoptotic or cell cycle inhibitory activities (104). Pre-clinical studies suggest that many compounds derived from natural products have potent activity against cancer cells, xenograft tumors, and that they can prevent carcinogenesis or metastasis of existing tumors (108). It is therefore not surprising that natural products are now being used for cancer prevention and/or therapy, and as adjuvants for conventional therapies. Although the results of most of the recent clinical studies have been disappointing, accumulating evidence suggests that combining these natural anti-inflammatory compounds with conventional therapies can lead to improved effects for patients.

For example, studies have shown that combining curcumin with vinorelbine or 5-fluorouracil leads to synergistic effects against cancer cells, while pretreatment of rats with curcumin prevents doxorubicin-associated cardiotoxicity (108). Ginseng saponins (ginsenosides) can also sensitize cancer cells to treatment with chemotherapeutic agents and protect against radiation-induced hematotoxicity (109). Similar protective and sensitization effects have been observed for several garlic compounds. One of the many anti-cancer garlic compounds, ajoene, enhanced the anti-proliferative and proapoptotic effects of the chemotherapeutic agents cytarabine and fludarbine against myeloid leukemia cells (110). Another garlic compound, Sallylmercaptocysteine, sensitized prostate cancer cells and xenograft tumors to treatment with docetaxel without any increase in toxicity to the mice (111).

It may also be possible to combine natural products with conventional anti-inflammatory agents in order to decrease their toxicity and enhance their activity. When carcinogen-dosed rats were fed a diet containing both curcumin and celecoxib, they developed fewer aberrant crypt foci compared to rats treated with either agent alone, and dramatically fewer foci compared to rats fed a control diet (112). Similarly, S-allylmercaptocysteine increased the anti-proliferative and pro-apoptotic effects of sulindac in human colon cancer cells (113). Thus, natural products can exert activities similar to conventional anti-inflammatory agents, and may have applications in preventing or treating human cancers by sensitizing cancer cells to conventional cancer therapies or protecting host cells from such treatment.

\section{iv. Other therapeutic agents targeting inflammation}

Another class of agents receiving a lot of attention for their anti-cancer and antiinflammatory effects is the statins. This class of agents was designed to act as inhibitors of HMGCoA reductase, thereby reducing cholesterol synthesis. However, the statins have proven to be active (based on epidemiological evidence and preclinical studies) against a variety of ailments, including hypertension, dementia, inflammation, and cancer (114). The compounds can be used to prevent or treat cancer as monotherapy, or can be used in combination with other anti-inflammatory agents or chemotherapeutic agents (5-fluorouracil, cisplatin, and doxorubicin) to yield more potent anticancer effects (115-117).

In addition, there are numerous other anticancer approaches that seek to modify the host immune response, decreasing anergy toward the tumor or decreasing the inflammatory microenvironment of the tumor. These strategies make use of Toll-like receptor agonists or antagonists (e.g. CpG oligonucleotides), antibodies or other agents that can decrease the expression or activity of pro-inflammatory molecules or their receptors (e.g. rituximab, zerumbone and CXCR4), or by treatment with specific cytokines or chemokines (e.g. IL-2 treatment for renal small cell carcinoma). Interested readers are directed to recent reviews about these topics $(118,121)$.

In brief, these illustrate just a few of the agents currently under investigation as the possible next generation of anti-inflammatory anti-cancer agents. While these compounds may eventually prove their utility, more emphasis should be placed on the agents currently approved for clinical use in order to bring about a benefit for patients in a shorter period of time.

\section{Conclusion}

Inflammation has been demonstrated to play a major role in the initiation, progression, and prognosis of cancer. The use of anti-inflammatory agents decreases the incidence and recurrence of various cancers, and can improve the prognosis for patients. Additionally, the use of anti-inflammatory agents in combination with conventional anti-cancer 
therapies is gaining ground, and is likely to yield many new therapeutic approaches to treating cancer within the next decade. However, a large body of research is still necessary to fully elucidate which lesions or cell types are amenable to cancer prevention or treatment with anti-inflammatory agents. Moreover, earlier diagnosis and treatment of inflammatory conditions (e.g. prostatitis, hepatitis, and pancreatitis) may be useful for preventing the initiation of cancer by inflammatory processes. While the currently used, FDA-approved, antiinflammatory agents undeniably have potent activities, their off-target effects and toxicities make them somewhat less attractive options for cancer therapy if used at their current doses and dosing frequencies. Thus, new combinations of agents, modifications to dosing or frequency, or the use of completely new anti-inflammatory compounds, may represent the next generation of care for both inflammatory diseases and cancers. Further research into the area is warranted.

\section{Acknowledgements}

This work was supported by NIH grants R01 CA112029 and R01 CA121211. E. Rayburn was supported by a DoD grant W81XWH-06-1-0063 and a T32 fellowship from the NIH/UAB Gene Therapy Center (CA075930). We apologize for not citing all the impressive publications by others in the field due to space limitations. We thank Drs. Hui Wang, Donald Hill, Mao Li, Wei Wang, and John Rinehart, other former and current members in our laboratory, and collaborators for their contributions to the research discussed in this article.

\section{References}

1. Rothman, I Stanford JL, Kuniyuki A, Berger RE. Selfreport of prostatitis and its risk factors in a random sample of middle-aged men. Urology 2004;64:876-9.

2. Nelson WG, De Marzo AM, DeWeese TL, Isaacs WB. The role of inflammation in the pathogenesis of prostate cancer. J Urol 2004;172:S6-11.

3. Rosenblatt KA, Wicklund KG, Stanford JL. Sexual factors and the risk of prostate cancer. Am J Epidemiol 2001;153:1152-8.

4. Adelstein P, Baldwin JA, Fedrich J. Cancers of the large bowel: associated disorders in individuals. Cancer 1979;43:2553-7.

5. Loftus EV, Jr. Epidemiology and risk factors for colorectal dysplasia and cancer in ulcerative colitis. Gastroenterol Clin North Am 2006;35:517-31.

6. Farrow B, Sugiyama Y, Chen A, Uffort E, Nealon W, Mark Evers B. Inflammatory mechanisms contributing to pancreatic cancer development. Ann Surg 2004;239:763-9.

7. Otsuki M. Chronic pancreatitis in Japan: epidemiology, prognosis, diagnostic criteria, and future problems. J Gastroenterol 2003;38:315-26.
8. Ammann RW, Akovbiantz A, Largiader F, Schueler G. Course and outcome of chronic pancreatitis. Longitudinal study of a mixed medical-surgical series of 245 patients. Gastroenterology 1984;86:820-8.

9. Strukov AI, Paukov VS, Orekhov OO. Morphology, pathogenesis and classification of interstitial lung diseases. Arkh Patol 1984;46:3-14.

10. Williams GT, Williams WJ. Granulomatous inflammation--a review. J Clin Pathol. 1983;36:723-33.

11. Hofseth LJ, Ying L. Identifying and defusing weapons of mass inflammation in carcinogenesis. Biochim Biophys Acta 2006;1765:74-84.

12. Sarkar D, Fisher PB. Molecular mechanisms of agingassociated inflammation. Cancer Lett. 2006;236:13-23.

13. Arias JI, Aller MA, Arias J. Cancer cell: using inflammation to invade the host. Mol Cancer 2007;6:29.

14. Coussens LM, Werb Z. Inflammation and cancer. Nature 2002;420:860-7.

15. Lu Y, Cai Z, Galson DL, Xiao G, Liu Y, George DE, et al. Monocyte chemotactic protein-1 (MCP-1) acts as a paracrine and autocrine factor for prostate cancer growth and invasion. Prostate 2006;66:1311-8.

16. Chavey C, Bibeau F, Gourgou-Bourgade S, Burlinchon $\mathrm{S}$, Boissière $\mathrm{F}$, Laune $\mathrm{D}$, et al. Oestrogen receptor negative breast cancers exhibit high cytokine content. Breast Cancer Res 2007;9:R15.

17. Van Waes C. Nuclear factor-kappaB in development, prevention, and therapy of cancer. Clin Cancer Res 2007;13:1076-82.

18. Rose-John S, Waetzig GH, Scheller J, Grötzinger J, Seegert D. The IL-6/sIL-6R complex as a novel target for therapeutic approaches. Expert Opin Ther Targets 2007;11:613-24.

19. Galliera E, Corsi MM, Bonecchi R, Locati M, Mantovani A. Chemokines as pharmacological targets. Mini Rev Med Chem 2008;8:638-46.

20. Knowles MA. Novel therapeutic targets in bladder cancer: mutation and expression of FGF receptors. Future Oncol 2008;4:71-83.

21. Waddell WR, Loughry RW. Sulindac for polyposis of the colon. J Surg Oncol 1983;24:83-87.

22. Kune GA. Kune S, Watson LF. Colorectal cancer risk, chronic illnesses, operations, and medications: case control results from the Melbourne Colorectal Cancer Study. Cancer Res 1988;48:4399-4404.

23. Wang D, Dubois RN. Prostaglandins and cancer. Gut 2006;55:115-22.

24. Lee JM Yanagawa J, Peebles KA, Sharma S, Mao JT, Dubinett SM. Inflammation in lung carcinogenesis: new targets for lung cancer chemoprevention and treatment. Crit Rev Oncol Hematol 2008;66:208-17.

25. Schonthal AH. Direct non-cyclooxygenase-2 targets of celecoxib and their potential relevance for cancer therapy. Br J Cancer 2007;97:1465-1468.

26. Zlotnik A. Involvement of chemokine receptors in organ-specific metastasis. Contrib Microbiol 2006;13:191-9. 27. Jana NR. NSAIDs and apoptosis. Cell Mol Life Sci. 2008;65:1295-301. 
28. de Groot DJ, de Vries EG, Groen HJ, de Jong S. Nonsteroidal anti-inflammatory drugs to potentiate chemotherapy effects: from lab to clinic. Crit Rev Oncol Hematol 2007;61:52-60.

29. Pari L, Tewas D, Eckel J. Role of curcumin in health and disease. Arch Physiol Biochem 2008;114:127-49.

30. Salman MC, Ayhan A. Use of anti-thrombotic agents during chemotherapy for epithelial ovarian cancer. Med Hypotheses 2006;66:1179-81.

31. Monnier Y, Zaric J, Rüegg C. Inhibition of angiogenesis by non-steroidal anti-inflammatory drugs: from the bench to the bedside and back. Curr Drug Targets Inflamm Allergy 2005;4:31-8.

32. Gridelli C, Gallo C, Ceribelli A, Gebbia V, Gamucci T, Ciardiello F, et al. Factorial phase III randomised trial of rofecoxib and prolonged constant infusion of gemcitabine in advanced non-small-cell lung cancer: the GEmcitabineCOxib in NSCLC (GECO) study. Lancet Oncol 2007;8:50012.

33. Arun B, Goss P. The role of COX-2 inhibition in breast cancer treatment and prevention. Semin Oncol $2004 ; 31: 22-9$

34. Smalley W, DuBois RN. Colorectal cancer and nonsteroidal anti-inflammatory drugs. Adv Pharmacol 1997;39:1-20.

35. Flossmann E, Rothwell PM; British Doctors Aspirin Trial and the UK-TIA Aspirin Trial. Effect of aspirin on long-term risk of colorectal cancer: consistent evidence from randomised and observational studies. Lancet 2007;369:1603-13

36. Bardia A, Ebbert JO, Vierkant RA, Limburg PJ, Anderson K, Wang AH, Olson JE, Vachon CM, Cerhan JR. Association of aspirin and nonaspirin nonsteroidal antiinflammatory drugs with cancer incidence and mortality. J Natl Cancer Inst 2007;99:881-9.

37. Zhang SM, Cook NR, Manson JE, Lee IM, Buring JE. Low-dose aspirin and breast cancer risk: results by tumour characteristics from a randomised trial. $\mathrm{Br} \mathrm{J}$ Cancer 2008;98:989-91.

38. Cruz-Correa M, Hylind LM, Romans KE, Booker SV, Giardiello FM. Long-term treatment with sulindac in familial adenomatous polyposis: a prospective cohort study. Gastroenterology 2002;122:641-5.

39. Gill S, Sinicrope FA. Colorectal cancer prevention: is an ounce of prevention worth a pound of cure? Semin Oncol 2005;32:24-34.

40. Harris RE, Chlebowski RT, Jackson RD, Frid DJ, Ascenseo JL, Anderson G, et al. Breast cancer and nonsteroidal anti-inflammatory drugs: prospective results from the Women's Health Initiative. Cancer Res 2003;63:6096-101.

41. Calaluce R, Earnest DL, Heddens D, Einspahr JG, Roe D, Bogert CL, et al. Effects of piroxicam on prostaglandin E2 levels in rectal mucosa of adenomatous polyp patients: a randomized phase IIb trial. Cancer Epidemiol Biomarkers Prev 2000;9:1287-92.

42. North GL. Celecoxib as adjunctive therapy for treatment of colorectal cancer. Ann Pharmacother 2001;35:1638-43.
43. Farley JH, Truong V, Goo E, Uyehara C, Belnap C, Larsen WI. A randomized double-blind placebo-controlled phase II trial of the cyclooxygenase- 2 inhibitor Celecoxib in the treatment of cervical dysplasia. Gynecol Oncol 2006;103:425-30.

44. Casanova I, Parreño M, Farré L, Guerrero S, Céspedes MV, Pavon MA, et al. Celecoxib induces anoikis in human colon carcinoma cells associated with the deregulation of focal adhesions and nuclear translocation of p130Cas. Int J Cancer 2006;118:2381-9.

45. www.clinicaltrials.gov

46. Witschi H, Espiritu I, Ly M, Uyeminami D. The chemopreventive effects of orally administered dexamethasone in Strain A/J mice following cessation of smoke exposure. Inhal Toxicol 2005;17:119-22.

47. Wang H, Li M, Rinehard JJ, Zhang R. Pretreatment with dexamethasone increases antitumor activity of carboplatin and gemcitabine in mice bearing human cancer xenografts: in vivo activity, pharmacokinetics, and clinical implications for cancer chemotherapy. Clin Cancer Res 2004;10:1633-44.

48. Wang H, Wang Y, Rayburn E, Hill DL, Rinehart JJ, Zhang R. Dexamethasone as a chemosensitizer for breast cancer chemotherapy: potentiation of the antitumor activity of adriamycin, modulation of cytokine expression, and pharmacokinetics. Int J Oncol 2007;30:947-53.

49. Arai Y, Nonomura N, Nakai Y, Nishimura K, Oka D, Shiba M, et al. The growth-inhibitory effects of dexamethasone on renal cell carcinoma in vivo and in vitro. Cancer Invest 2008;26:35-40.

50. Gong H, Jarzynka MJ, Cole TJ, Lee JH, Wada T, Zhang B, et al. Glucocorticoids antagonize estrogens by glucocorticoid receptor-mediated activation of estrogen sulfotransferase. Cancer Res 2008;68:7386-93.

51. Yano A, Fujii Y, Iwai A, Kageyama Y, Kihara K. Glucocorticoids suppress tumor angiogenesis and in vivo growth of prostate cancer cells. Clin Cancer Res. 2006;12:3003-9.

52. McLean JS, Frame MC, Freshney RI, Vaughan PF, Mackie AE, Singer I. Phenotypic modification of human glioma and non-small cell lung carcinoma by glucocorticoids and other agents. Anticancer Res 1986;6:1101-6.

53. Fecker LF, Stockfleth E, Nindl I, Ulrich C, Forschner $\mathrm{T}$, Eberle J. The role of apoptosis in therapy and prophylaxis of epithelial tumours by nonsteroidal antiinflammatory drugs (NSAIDs). Br J Derm 2007;156:25-33. 54. Albini A, Tosetti F, Benelli R, Noonan DM. Tumor inflammatory angiogenesis and its chemoprevention. Cancer Res 2005;65:10637-41.

55. Sawaoka H, Tsuji S, Tsujii M, Gunawan ES, Sasaki Y, Kawano S, et al. Cyclooxygenase inhibitors suppress angiogenesis and reduce tumor growth in vivo. Lab Invest 1999;79:1469-77.

56. Lev-Ari S, Lichtenberg D, Arber N. Compositions for treatment of cancer and inflammation. Recent Patents Anti-Cancer Drug Discov 2008;3:55-62.

57. Albouy B, Tourani JM, Allain P, Rolland F, Staerman F, Eschwege P, et al. Preliminary results of the Prostacox 
phase II trial in hormonal refractory prostate cancer. BJU Int 2007;100:770-4.

58. Javle MM, Cao S, Durrani FA, Pendyala L, Lawrence DD, Smith PF, et al. Celecoxib and mucosal protection: translation from an animal model to a phase I clinical trial of celecoxib, irinotecan, and 5-fluorouracil. Clin Cancer Res 2007;13:965-71.

59. Fabi A, Metro G, Papaldo P, Mottolese M, Melucci E, Carlini P. Impact of celecoxib on capecitabine tolerability and activity in pretreated metastatic breast cancer: results of a phase II study with biomarker evaluation. Cancer Chemother Pharmacol 2008;64:717-25.

60. Wang H, Li M, Rinehart JJ, Zhang R. Dexamethasone as a chemoprotectant in cancer chemotherapy: hematoprotective effects and altered pharmacokinetics and tissue distribution of carboplatin and gemcitabine. Cancer Chemother Pharmacol 2004;53:459-67.

61. Leggas M, Kuo KL, Robert F, Cloud G, Deshazo M, Zhang $\mathrm{R}$, et al. Intensive anti-inflammatory therapy with dexamethasone in patients with non-small cell lung cancer: effect on chemotherapy toxicity and efficacy. Cancer Chemother Pharmacol 2008; (epub ahead of print).

62. Karthaus M, Ballo H, Abenhardt W, Steinmetz T, Geer T, Schimke J, et al. Prospective, double-blind, placebo-controlled, multicenter, randomized phase III study with orally administered budesonide for prevention of irinotecan (CPT-11)-induced diarrhea in patients with advanced colorectal cancer. Oncology 2005;68:326-32.

63. Candelario-Jalil E, Taheri S, Yang Y, Sood R, Grossetete M, Estrada EY, et al. Cyclooxygenase inhibition limits blood-brain barrier disruption following intracerebral injection of tumor necrosis factor-alpha in the rat. J Pharmacol Exp Ther 2007;323:488-98.

64. Kim H, Lee JM, Park JS, Jo SA, Kim YO, Kim CW, et al. Dexamethasone coordinately regulates angiopoietin-1 and VEGF: a mechanism of glucocorticoid-induced stabilization of blood-brain barrier. Biochem Biophys Res Commun 2008;372:243-8.

65. Karjalainen MJ, Neuvonen PJ, Backman JT. In vitro inhibition of CYP1A2 by model inhibitors, antiinflammatory analgesics and female sex steroids: predictability of in vivo interactions. Basic Clin Pharmacol Toxicol. 2008;103:157-165.

66. Hersh EV, Pinto A, Moore PA. Adverse drug interactions involving common prescription and over-thecounter analgesic agents. Clin Ther 2007;29:2477-97.

67. Wang L-C, Ching LM, Paxton JW, Kestell P, Sutherland R, Zhuang L, et al. Enhancement of the action of the antivascular drug 5,6-dimethylxanthenone-4-acetic acid (DMXAA; ASA404) by non-steroidal antiinflammatory drugs. Investig New Drugs 2008;epub ahead of print

68. Heldin CH, Rubin K, Pietras K, Ostman A. High interstitial fluid pressure - an obstacle in cancer therapy. Nat Rev Cancer 2004;4:806-13.

69. Raju B, Haug SR, Ibrahim SO, Heyeraas KJ. High interstitial fluid pressure in rat tongue cancer is related to increased lymph vessel area, tumor size, invasiveness and decreased body weight. J Oral Pathol Med 2008;37:137-44.
70. Kristjansen PE, Boucher Y, Jain RK. Dexamethasone reduces the interstitial fluid pressure in a human colon adenocarcinoma xenograft. Cancer Res 1993;53:4764-66.

71. Herrera FG, Chan P, Doll C, Milosevic M, Oza A, Syed A, et al. A prospective phase I-II trial of the cyclooxygenase-2 inhibitor celecoxib in patients with carcinoma of the cervix with biomarker assessment of the tumor microenvironment. Int $\mathrm{J}$ Radiat Oncol Biol Phys 2007;67:97-103.

72. Ponthan F, Wickström M, Gleissman H, Fuskevåg OM, Segerström L, Sveinbjörnsson B, et al. Celecoxib prevents neuroblastoma tumor development and potentiates the effect of chemotherapeutic drugs in vitro and in vivo. Clin Cancer Res 2007;13:1036-44.

73. Dandekar DS, Lopez M, Carey RI, Lokeshwar BL. Cyclooxygenase-2 inhibitor celecoxib augments chemotherapeutic drug-induced apoptosis by enhancing activation of caspase -3 and -9 in prostate cancer cells. Int J Cancer 2005;115:484-92.

74. Nakata E, Mason KA, Hunter N, Husain A, Raju U, Liao $\mathrm{Z}$, et al. Potentiation of tumor response to radiation or chemoradiation by selective cyclooxygenase- 2 enzyme inhibitors. Int J Radiat Oncol Biol Phys 2004;58:369-75

75. Stark LA, Din FV, Zwacka RM, Dunlop MG. Aspirininduced activation of the NF-kappaB signaling pathway: a novel mechanism for aspirin-mediated apoptosis in colon cancer cells. FASEB J 2001;7:1273-75.

76. Loveridge CJ, MacDonald AD, Thoms HC, Dunlop MG, Stark LA. The proapoptotic effects of sulindac, sulindac sulfone and indomethacin are mediated by nucleolar translocation of the RelA(p65) subunit of NFkappaB. Oncogene 2008;27:2648-55.

77. Van Wijngaarden J, van Beek E, van Rossum G, van der Bent C, Hoekman K, van der Pluijm G, et al. Celecoxib enhances doxorubicin-induced cytotoxicity in MDA-MB231 cells by NF-kappaB-mediated increase of intracellular doxorubicin accumulation. Eur J Cancer 2007;43:433-42.

78. Castro-Caldas M, Mendes AF, Duarte CB, Lopes MC. Dexamethasone-induced and estradiol-induced CREB activation and annexin 1 expression in CCRF-CEM lymphoblastic cells: evidence for the involvement of cAMP and p38 MAPK. Mediators Inflamm 2003;12:37-46.

79. Gu Q, Wang JD, Xia HH, Lin MC, He H, Zou B, et al. Activation of the caspase-8/Bid and Bax pathways in aspirin-induced apoptosis in gastric cancer. Carcinogenesis 2005;26:541-45.

80. Pan MR, Chang HC, Hung WC. Non-steroidal antiinflammatory drugs suppress the ERK signaling pathway via block of Ras/c-Raf interaction and activation of MAP kinase phosphatases. Cell Signal 2008;20:1134-41.

81. Chen XJ, Xiao W, Qu X, Zhou SY. NS-398 enhances the efficacy of gemcitabine against lung adenocarcinoma through up-regulation of p21WAF1 and p27KIP1 protein. Neoplasma 2008;55:200-204.

82. Han A, Song Z, Tong C, Hu D, Bi X, Augenlicht LH, et al. Sulindac suppresses beta-catenin expression in human cancer cells. Eur J Pharmacol 2008;583:26-31. 
83. Dikshit P, Chatterjee M, Goswami A, Mishra A, Jana NR. Aspirin induces apoptosis through the inhibition of proteasome function. J Biol Chem 2006;281:29228-35.

84. Dittmann KH, Mayer C, Ohneseit PA, Raju U, Andratschke NH, Milas L, et al. Celecoxib induced tumor cell radiosensitization by inhibiting radiation induced nuclear EGFR transport and DNA-repair: a COX-2 independent mechanism. Int J Radiat Oncol Biol Phys 2008;70:203-12.

85. Chuang HC, Kardosh A, Gaffney KJ, Petasis NA, Schönthal AH. COX-2 inhibition is neither necessary nor sufficient for celecoxib to suppress tumor cell proliferation and focus formation in vitro. Mol Cancer 2008;7:38.

86. O'Connor R, O'Leary M, Ballot J, Collins CD, Kinsella $\mathrm{P}$, Mager DE. et al. A phase I clinical and pharmacokinetic study of the multi-drug resistance protein-1 (MRP-1) inhibitor sulindac, in combination with epirubicin in patients with advanced cancer. Cancer Chemother Pharmacol 2007;59:79-87.

87. Zrieki A, Farinotti R, Buyse M. Cyclooxygenase inhibitors down regulate P-glycoprotein in human colorectal Caco-2 cell line. Pharm Res 2008;25:1991-01.

88. Gradilone A, Pulcinelli FM, Lotti LV, Trifirò E, Martino S, Gandini O, et al. Celecoxib upregulates multidrug resistance proteins in colon cancer: lack of synergy with standard chemotherapy. Curr Cancer Drug Targets 2008;8:414-20.

89. Fleming MT, Morris MJ, Heller G, Scher HI. Posttherapy changes in PSA as an outcome measure in prostate cancer clinical trials. Nat Clin Pract Oncol 2006;3:658-667.

90. Wang H, Li M, Lin W, Wang W, Zhang Z, Rayburn ER, et al. Extracellular activity of cyclic AMP-dependent protein kinase as a biomarker for human cancer detection: distribution characteristics in a normal population and cancer patients. Cancer Epidemiol Biomarkers Prev 2007;16:789-95.

91. Stoll BA. Dexamethasone in advanced breast cancer. Cancer 1960;13:1074-80.

92. Galicich JH, French LA. Use of dexamethasone in the treatment of cerebral edema resulting from brain tumors and brain surgery. Am Pract Dig Treat 1961;12:169-74.

93. Belman S, Troll W. The inhibition of croton oilpromoted mouse skin tumorigenesis by steroid hormones. Cancer Res 1972;32:450-4.

94. Urtasun RC, Tanasichuk H, Fulton D, Agboola O, Turner AR, Koziol D, et al. High dose misonidazole with dexamethasone rescue: a possible approach to circumvent neurotoxicity. Int J Radiat Oncol Biol Phys 1982;8:365-9.

95. Bakowski MT. Advances in anti-emetic therapy. Cancer Treat Rev 1984;11:237-56.

96. Schiffmann S, Maier TJ, Wobst I, Janssen A, CorbanWilhelm H, Angioni C, et al. Biochem Pharmacol 2008;76:179-87.

97. Schonthal AH, Chen TC, Hofman FM, Louie SG, Petasis NA. Celecoxib analogs that lack COX-2 inhibitory function: preclinical development of novel anticancer drugs. Expert Opin Investig Drugs 2008;17:197-08.
98. Ding H, Han C, Guo D, Wang D, Duan W, Chen CS, et al. Sensitivity to the non-COX inhibiting celecoxib derivative, OSU03012, is p21(WAF1/CIP1) dependent. Int J Cancer 2008;123:2931-38.

99. Bandarage UK, Janero DR. Nitric oxide-releasing nonsteroidal anti-inflammatory drugs: novel gastrointestinal-sparing drugs. Mini Rev Med Chem 2001;1:57-70.

100.Bandarage UK, Chen L, Fang X, Garvey DS, Glavin A Janero DR, et al. Nitrosothiol esters of diclofenac: synthesis and pharmacological characterization as gastrointestinal-sparing prodrugs. J Med Chem 2000;43:4005-16

101.Kartasasmita RE, Laufer S, Lehmann J. NO-donors (VII (1)): synthesis and cyclooxygenase inhibitory properties of $\mathrm{N}$-and S-nitrooxypivaloyl-cysteine derivatives of naproxen -- a novel type of NO-NSAID. Arch Pharm 2002;335:363-6.

102.Kashfi K, Ryan Y, Qiao LL, Williams JL, Chen J, Del Soldato P, Traganos F, et al. Nitric oxide-donating nonsteroidal anti-inflammatory drugs inhibit the growth of various cultured human cancer cells: evidence of a tissue type-independent effect. J Pharmaco Exp Ther 2002;303:1273-82.

103.Rigas B, Williams JL. NO-donating NSAIDs and cancer: an overview with a note on whether $\mathrm{NO}$ is required for their action. Nitric Oxide 2008;19:199-04.

104.Aggarwal BB, Shishodia S. Molecular targets of dietary agents for prevention and therapy of cancer. Biochem Pharmacol 2006;71:1397-421.

105.Das S, Das DK. Anti-inflammatory responses of resveratrol. Inflamm Allergy Drug Targets 2007;6:168-73.

106. Anand P, Sundaram C, Jhurani S, Kunnumakkara AB, Aggarwal BB. Curcumin and cancer: an "old-age" disease with an "age-old" solution. Cancer Lett 2008;267:133-64.

107.Hofseth LJ, Wargovich MJ. Inflammation, cancer, and targets of ginseng. J Nutr 2007;137:183S-185S.

108. Strimpakos AS, Sharma RA. Curcumin: preventive and therapeutic properties in laboratory studies and clinical trials. Antioxid Redox Signal 2008;10:511-45.

109. Helms S. Cancer prevention and therapeutics: Panax ginseng. Alternative Med Rev 2004;9:259-274

110.Hassan HT. Ajoene (natural garlic compound): a new anti-leukaemia agent for AML therapy. Leuk Res 2004;28:667-71.

111. Howard EW, Lee DT, Chiu YT, Chua CW, Wang X, Wong YC. Evidence of a novel docetaxel sensitizer, garlicderived S-allylmercaptocysteine, as a treatment option for hormone refractory prostate cancer. Int $\mathrm{J}$ Cancer 2008;122:1941-8.

112.Shpitz B, Giladi N, Sagiv E, Lev-Ari S, Liberman E, Kazanov D, et al. Celecoxib and curcumin additively inhibit the growth of colorectal cancer in a rat model. Digestion 2006;74:140-4.

113. Shirin H, Pinto JT, Kawabata Y, Soh JW, Delohery T, Moss SF, et al. Antiproliferative effects of Sallylmercaptocysteine on colon cancer cells when tested 
alone or in combination with sulindac sulfide. Cancer Res 2001;61:725-31.

114.Paraskevas KI, Tzovaras AA, Briana DD, Mikhailidis DP. Emerging indications for statins: a pluripotent family of agents with several potential applications. Curr Pharm Des 2007;13:3622-36.

115.Katz MS. Therapy insight: Potential of statins for cancer chemoprevention and therapy. Nat Clin Pract Oncol 2005;2:82-9.

116.Sassano A, Platanias LC. Statins in tumor suppression. Cancer Lett 2008;260:11-19.

117.Xiao H, Yang CS. Combination regimen with statins and NSAIDs: a promising strategy for cancer chemoprevention. Int J Cancer 2008;123:983-90.

118. Meyer T, Stockfleth E. Clinical investigations of Tolllike receptor agonists. Expert Opin Investig Drugs 2008;17:1051-65.

119.Yu L, Chen S. Toll-like receptors expressed in tumor cells: targets for therapy. Cancer Immunol Immunother 2008;57:1271-8.

120.Sung B, Jhurani S, Ahn KS, Mastuo Y, Yi T, Guha S, et al. Jhurani S, Ahn KS, Mastuo Y, Yi T, Guha S Cancer Res 2008;68:8938-44.

121. Coppin C. Immunotherapy for renal cell cancer in the era of targeted therapy. Expert Rev Anticancer Ther 2008;8:907-19. 